\title{
TAIL ASYMPTOTICS OF THE SUPREMUM OF A REGENERATIVE PROCESS
}

\author{
ZBIGNIEW PALMOWSKI, ${ }^{*}$ Utrecht University and University of Wroctaw \\ BERT ZWART, ${ }^{* *}$ Georgia Institute of Technology
}

\begin{abstract}
We give precise asymptotic estimates of the tail behavior of the distribution of the supremum of a process with regenerative increments. Our results cover four qualitatively different regimes involving both light tails and heavy tails, and are illustrated with examples arising in queueing theory and insurance risk.
\end{abstract}

Keywords: Cramér condition; fluid model; perturbed random walk; regenerative process; ruin probability; subexponential distribution

2000 Mathematics Subject Classification: Primary 60F10; 60K25; 62P05

\section{Introduction}

Regenerative processes are a versatile tool in stochastic modeling, as they are general enough to cover many applications and at the same time provide a natural and tractable extension of the random walk. In particular, the computation of overflow probabilities in (fluid) queues and ruin probabilities in insurance can often be reduced to the study of the maximum of a process of which the increments are regenerative. Specifically, let $S(t), t \geq 0$, be a càdlàg process almost surely drifting to $-\infty$, such that $S(0)=0$. Suppose that there exists a renewal process with renewal epochs $T_{i}, 0 \leq T_{0}<T_{1}<\cdots$, such that

$$
(S(t))_{0 \leq t<T_{0}}, \quad\left(S\left(T_{0}+t\right)-S\left(T_{0}\right)\right)_{0 \leq t<T_{1}-T_{0}}, \quad \cdots
$$

are independent and the distributions of $\left(S\left(T_{k}+t\right)-S\left(T_{k}\right)\right)_{0 \leq t<T_{k+1}-T_{k}}, k \geq 0$, are identical. We call $T_{n}, n \geq 0$, the regeneration or renewal epochs of $S(t), t \geq 0$. If $T_{0}=0$, we say that $(S(t))$ is zero-delayed. Define

$$
M=\sup _{t \geq 0} S(t), \quad M_{n+1}=\sup _{T_{n} \leq t<T_{n+1}} S(t)-S\left(T_{n}\right), \quad n \geq 0,
$$

and let $X_{n+1}=S\left(T_{n+1}\right)-S\left(T_{n}\right)$ and $S_{n}=S\left(T_{n}\right)$, for $n \geq 0$. This is strongly related to the setting considered in [3] and many other papers. Typically, the distribution of $M$ is too complicated to compute exactly. Therefore, we are often concerned with the tail behavior of $M$, i.e. the behavior of $\mathrm{P}\{M>x\}$ as $x$ grows large. This forms the motivation for the present paper.

In this paper we focus on the zero-delayed case, and thus have the identity

$$
M=\sup _{n \geq 1}\left[S_{n-1}+M_{n}\right]
$$

Received 20 April 2006; revision received 11 February 2007.

* Postal address: Mathematical Institute, University of Wrocław, pl. Grunwaldzki 2/4, 50-384 Wrocław, Poland. Email address: zpalma@math.uni.wroc.pl

** Postal address: H. Milton Stewart School of Industrial and Systems Engineering, Georgia Institute of Technology, 765 Ferst Drive, Atlanta, GA 30332-0205, USA. Email address: bertzwart@gatech.edu 
The sequence $M_{n}, n \geq 1$, is independent and identically distributed but depends on the random walk $S_{n}, n \geq 1$, since $M_{n}$ and $X_{n}$ are dependent. Note that the sequence of pairs $\left(M_{n}, X_{n}\right), n \geq$ 1 , is independent and identically distributed. Thus, the regenerative setting can be viewed as a special case of the more general framework of the perturbed random walk, which was considered in a recent paper by Araman and Glynn [1]. They investigated the tail behavior of $M$ in a variety of cases.

The main goal of this work is to analyze the tail behavior of $M$ in the perturbed random walk setting (1), under conditions that are general enough to be applicable to regenerative processes. For random walk maxima, it is well known (see, e.g. [4], [10], and [20]) that the description of the tail behavior can be classified into three main regimes: (i) the Cramér case, (ii) the intermediate case, and (iii) the heavy tail case. Our main results cover these cases for perturbed random walks. In addition, we identify a fourth main case, in which the perturbations dominate the tail behavior of $M$. Specifically, our results are as follows:

- The first scenario we consider is when the Cramér condition holds for $X_{1}$, i.e. we assume that there exists a strictly positive solution $\kappa$ to the equation $\mathrm{E}\left\{\mathrm{e}^{\kappa X_{1}}\right\}=1$. In addition, we assume that the tail of $M_{1}$ is not too heavy (in a sense we make precise later on). These assumptions allow us to apply the implicit renewal theory developed by Goldie [14], to obtain the tail behavior of $M$. The results of [14] have mostly been applied to autoregressive processes but have much wider applicability. Special cases of our result have been derived before, by Araman and Glynn [1] and by Schmidli [25]. We note that the main result of [1], which covers the case in which the perturbations $\left(M_{n}\right)$ form a stationary sequence independent of the random walk $\left(S_{n}\right)$, is not covered in the present paper.

- A qualitatively different case occurs when $M_{1}$ is light tailed but heavier tailed than $\sup _{n} S_{n}$. Again we exploit theory developed for autoregressive processes. In particular, we utilize stochastic ordering arguments proposed in [16] to extend and unify both Theorem 3 of [1] and Example 2 of [16].

- We again apply stochastic ordering arguments to analyze the intermediate case, which also pertains in the case of a standard random walk. We derive the tail behavior of $M$ under the assumption that the right tail of $X_{1}$ is in the class $\delta(\alpha)$, and that the right tail of $M_{1}$ is not heavier than the right tail of $X_{1}$.

- A fourth regime we consider is that in which $M_{1}^{*}=\max \left\{M_{1}, X_{1}\right\}$ has a heavy tail in a sense we make precise later on. This case has been investigated before, in [1], under the additional assumption that $X_{1}$ is light tailed. We also extend a result of [3], where it was assumed that the tails of $M_{1}^{*}$ and $X_{1}$ asymptotically coincide (note that our $M_{1}^{*}$ is identical to their $M_{1}$ ). Other related papers are [12] and [13], where a class of modulated random walks and Lévy processes with heavy-tailed increments were considered.

We illustrate our results by considering some specific models that arise in telecommunications and insurance. We first consider a basic on-off fluid model where $S(t), t \geq 0$, either increases with rate $r-c>0$, or decreases with rate $c$. Then we investigate an insurance risk model. More precisely, we consider a Cox-type process which was introduced and motivated in [26]. We apply our general result to derive an exponential estimate for the ruin probability, and obtain bounds on the prefactor. 
The rest of the paper is organized as follows. Section 2 contains the main results: the four different cases are investigated in Subsections 2.1-2.4. Section 3 is devoted to the on-off model. The insurance risk model is investigated in Section 4. In Section 5 we conclude.

\section{General results}

In this section we present the main results of this work. We focus on four cases: first, we consider the situation in which $M_{1}^{*}$ is heavy tailed, in Section 2.1. Then we assume that both $X_{1}$ and $M_{1}$ are light tailed. Three further distinctions arise here, and are respectively treated in Subsections 2.2, 2.3, and 2.4.

We always assume that the joint distribution of $\left(X_{1}, M_{1}\right)$ satisfies $\mathrm{E}\left\{X_{1}\right\} \in(-\infty, 0)$, $\mathrm{E}\left\{M_{1}\right\}<\infty$, and $\mathrm{P}\left\{M_{1}=-\infty\right\}=0$. The last assumption is not restrictive: for regenerative processes we have the representation $M_{1}=\sup _{t \in\left[0, T_{1}\right)} S(t) \geq 0$. Throughout this section we use various standard results for the class $\mathcal{L}$ of long-tailed distributions, the class $\delta$ of subexponential distributions, and the class $\delta(\gamma), \gamma \geq 0$. A standard reference on such distributions is the textbook [11]. For two functions $f(x)$ and $g(x)$, we write $f(x) \sim g(x)$ if $f(x)=g(x)(1+o(1))$ as $x \rightarrow \infty$.

\subsection{The heavy tail case}

Our first result concerns the case in which $M_{n}^{*}=\max \left\{X_{n}, M_{n}\right\}$ is heavy tailed.

Theorem 1. If $\mathrm{E}\left\{M_{1}^{*}\right\}<\infty$ and $\min \left\{1, \int_{x}^{\infty} \mathrm{P}\left\{M_{1}^{*}>u\right\} \mathrm{d} u\right\}$ is subexponential, then

$$
\mathrm{P}\{M>x\} \sim \frac{1}{\mu} \int_{x}^{\infty} \mathrm{P}\left\{M_{1}^{*}>u\right\} \mathrm{d} u
$$

as $x \rightarrow \infty$, with $\mu=-\mathrm{E}\left\{X_{1}\right\}$.

This is an extension of Theorem 3.3 of [2], where it was assumed that $\mathrm{P}\left\{M_{1}^{*}>x\right\} \sim$ $\mathrm{P}\left\{X_{1}>x\right\}$. In Section 3 we give examples to show that this condition is sometimes too restrictive. Theorem 1 is also related to Theorem 4 of [1]. There it was assumed that $X_{1}$ is light tailed and that the marginal distribution of $M_{1}$ has a hazard rate converging to 0 .

Proof of Theorem 1. The proof consists in deriving lower and upper bounds which asymptotically coincide.

We start with the lower bound, for which we adapt a standard (see [3], [13], [12], and [28]) technique to our setting. The idea is to identify a way in which the event $\{M>x\}$ occurs. Informally speaking, we choose an event on which $S_{n-1}-M_{n}, n \geq 1$, behaves in a typical way up to some time $k$ for which $M_{k+1}^{*}=\max \left\{M_{k+1}, X_{k+1}\right\}$ is large. By also including the event that $M_{k+2}$ is not too small, we ensure that $M>x$.

Let $\delta, 0<\delta<\mu$, be given, and for $n \geq 1$ define the event $E_{n} \equiv E_{n}(\delta, K)$ by

$$
E_{n}=\left\{S_{k} \in(-k(\mu+\delta)-K,-k(\mu-\delta)+K), k \leq n\right\} .
$$

In addition, consider the event $F_{n} \equiv F_{n}(\delta, K)$, defined by

$$
F_{n}=\left\{M_{k}<\delta k+K, k \leq n\right\} .
$$

Also define $G(x)=\mathrm{P}\left\{M_{n}<x\right\}=\mathrm{P}\left\{M_{1}<x\right\}$, and let $K$ be such that $\bar{G}(K)=1-G(K)<\frac{1}{2}$. Since $\log (1-x) \geq-2 x$ if $x \in\left(0, \frac{1}{2}\right)$, we see that

$$
\log \mathrm{P}\left\{F_{n}\right\}=\sum_{k=1}^{n} \log (1-\bar{G}(\delta k+K)) \geq-2 \sum_{k=1}^{\infty} \bar{G}(\delta k+K)=-2 \mathrm{E}\left\{\left\lfloor\left(M_{1}-K\right)^{+} / \delta\right\rfloor\right\},
$$


where $y^{+}=\max \{y, 0\}$. Since $\mathrm{E}\left\{M_{1}\right\}<\infty$, the last expression converges to 0 if $K \rightarrow \infty$ for any $\delta>0$. Combining this fact with the weak law of large numbers for $S_{n}, n \geq 1$, we arrive at the following conclusion: for every $\varepsilon>0$ there exists a $K$ such that $\mathrm{P}\left\{E_{n} \cap F_{n}\right\} \geq 1-\varepsilon$.

For $n \geq 1$, we define the event

$$
G_{n}=F_{n} \cap E_{n} \cap\left\{M_{n+1}^{*}>x+n(\delta+\mu)+2 K\right\} \cap\left\{M_{n+2}>-K\right\} .
$$

Observe that the events $G_{n}, n \geq 1$, are disjoint and that $G_{n}$ implies $\{M>x\}$ for every $n \geq 1$. Consequently,

$$
\begin{aligned}
\mathrm{P}\{M>x\} & \geq \mathrm{P}\left\{\bigcup_{n=1}^{\infty} G_{n}\right\}=\sum_{n=1}^{\infty} \mathrm{P}\left\{G_{n}\right\} \\
& \geq(1-\varepsilon) \sum_{n=1}^{\infty} \mathrm{P}\left\{M_{n+1}^{*}>x+n(\delta+\mu)+2 K\right\} \mathrm{P}\left\{M_{n+2}>-K\right\} \\
& \sim \frac{1-\varepsilon}{\delta+\mu} \mathrm{P}\left\{M_{1}>-K\right\} \int_{x+K}^{\infty} \mathrm{P}\left\{M_{1}^{*}>u\right\} \mathrm{d} u \\
& \sim \frac{1-\varepsilon}{\delta+\mu} \mathrm{P}\left\{M_{1}>-K\right\} \int_{x}^{\infty} \mathrm{P}\left\{M_{1}^{*}>u\right\} \mathrm{d} u
\end{aligned}
$$

where in the last two steps we have used the fact that $M_{1}^{*}$ is long tailed. This implies that

$$
\liminf _{x \rightarrow \infty} \frac{\mathrm{P}\{M>x\}}{\int_{x}^{\infty} \mathrm{P}\left\{M_{1}^{*}>u\right\} \mathrm{d} u} \geq \frac{1-\varepsilon}{\delta+\mu} \mathrm{P}\left\{M_{1}>-K\right\} .
$$

The proof of the lower bound follows by letting $K \rightarrow \infty$ and $\delta, \varepsilon \downarrow 0$.

To obtain an asymptotic upper bound, let $y>0$ be given and construct the random walk $S_{n}^{y}, n \geq 0$, with $S_{0}^{y}=0$. For $k \geq 1$, let $X_{k}^{y}=X_{k}$ if $M_{k}^{*} \leq y$ and let $X_{k}^{y}=M_{k}^{*}$ if $M_{k}^{*}>y$. Finally, let $S_{n}^{y}=X_{1}^{y}+\cdots+X_{n}^{y}, n \geq 1$. Informally, the increments of the random walk $S_{n}^{y}, n \geq 0$, are the same as those of $S_{n}, n \geq 0$, except when a large value of $M_{n}^{*}$ occurs.

Obviously $S_{n} \leq S_{n}^{y}$ for any $y>0$ and $n \geq 1$. Moreover, we have the following crucial bound:

$$
\sup _{n \geq 1}\left[S_{n-1}+M_{n}\right] \leq \sup _{n \geq 0} S_{n}^{y}+y
$$

For $x>y$, we have $\mathrm{P}\left\{X_{k}^{y}>x\right\}=\mathrm{P}\left\{M_{k}^{*}>x\right\}$, which implies that the integrated tail of $X_{k}^{y}$ is subexponential. Thus, we can apply Veraverbeke's theorem (see, e.g. [27] or [28]), yielding

$$
\mathrm{P}\left\{\sup _{n \geq 1} S_{n}^{y}>x\right\} \sim \frac{1}{-\mathrm{E}\left\{X_{1}^{y}\right\}} \int_{x}^{\infty} \mathrm{P}\left\{M_{1}^{*}>u\right\} \mathrm{d} u .
$$

Putting everything together, we conclude that

$$
\limsup _{x \rightarrow \infty} \frac{\mathrm{P}\{M>x\}}{\int_{x}^{\infty} \mathrm{P}\left\{M_{1}^{*}>u\right\} \mathrm{d} u} \leq \limsup _{x \rightarrow \infty} \frac{\mathrm{P}\left\{\sup _{n \geq 1} S_{T_{n}}^{y}>x-y\right\}}{\int_{x}^{\infty} \mathrm{P}\left\{M_{1}^{*}>u\right\} \mathrm{d} u} \leq \frac{1}{-\mathrm{E}\left\{X_{1}^{y}\right\}} .
$$

By dominated convergence, it follows that $-\mathrm{E}\left\{X_{1}^{y}\right\} \rightarrow \mu$ as $y \rightarrow \infty$. 


\subsection{Dominating perturbations}

We now suppose that $M_{1}$ is light tailed. More precisely, we assume that

$$
\mathrm{P}\left\{M_{1}>x\right\}=m_{1}(x) \mathrm{e}^{-v x},
$$

with $m_{1}(\cdot)$ such that $m_{1}(x+y) \sim m_{1}(x)$ as $x \rightarrow \infty$, for any fixed $y$. This is equivalent to the requirement that the right tail of the distribution of $\exp \left\{M_{1}\right\}$ is regularly varying with index $-v$.

To formulate our result, we need to make two more assumptions. The first additional assumption we invoke is that

$$
\mathrm{E}\left\{\mathrm{e}^{\nu X_{1}}\right\}<1 .
$$

Our final assumption is of a more technical nature: let $\tilde{X}_{1}$ be an independent copy of $X_{1}$ which is also independent of $M_{1}$, and suppose that

$$
\mathrm{P}\left\{\tilde{X}_{1}+M_{1}>x\right\} \sim \mathrm{E}\left\{\mathrm{e}^{\nu X_{1}}\right\} \mathrm{P}\left\{M_{1}>x\right\} .
$$

This assumption is a consequence of (2), (3) and a minor additional regularity condition. In particular, one of the following three conditions suffices: (i) $\mathrm{E}\left\{\mathrm{e}^{\eta X_{1}}\right\}<\infty$ for some $\eta>v$ (Breiman's theorem), (ii) $\lim _{x \rightarrow \infty} m_{1}(x)>0$, or (iii) $m_{1}(x)$ is decreasing and in $8^{*}$; see [9] for details. Moreover, assumption (4) implies that $\mathrm{P}\left\{X_{1}>x\right\}=o\left(\mathrm{P}\left\{M_{1}>x\right\}\right)$.

The following result extends and unifies Example 2 of [16] (where condition (i) was assumed), and Theorem 4 of [1] (where it was assumed that $\lim _{x \rightarrow \infty} m_{1}(x)>0$ ). To prove our result, we adapt the arguments in [16] to our setting.

Theorem 2. If (2)-(4) hold, then

$$
\mathrm{P}\{M>x\} \sim \frac{1}{1-\mathrm{E}\left\{\mathrm{e}^{\nu X_{1}}\right\}} \mathrm{P}\left\{M_{1}>x\right\} .
$$

In the remainder of this subsection we assume that (2)-(4) are in force. The proof of Theorem 2 involves the following lemma.

Lemma 1. Suppose that $Y \geq 0$ is independent of $\left(M_{1}, X_{1}\right)$ and that

$$
\mathrm{P}\{Y>x\} \sim c_{Y} \mathrm{P}\left\{M_{1}>x\right\}
$$

for a constant $c_{Y} \in(0, \infty)$. Then $\mathrm{P}\left\{\max \left\{M_{1}, X_{1}+Y\right\}>x\right\} \sim\left(1+c_{Y} \mathrm{E}\left\{\mathrm{e}^{v X_{1}}\right\}\right) \mathrm{P}\left\{M_{1}>x\right\}$.

Proof. An asymptotic upper bound simply follows from the assumptions, the bound

$$
\mathrm{P}\left\{\max \left\{M_{1}, X_{1}+Y\right\}>x\right\} \leq \mathrm{P}\left\{M_{1}>x\right\}+\mathrm{P}\left\{X_{1}+Y>x\right\},
$$

and the fact that (4) is closed under tail equivalence. To prove that this upper bound is tight, it suffices to show that $\mathrm{P}\left\{\min \left\{M_{1}, X_{1}+Y\right\}>x\right\}=o\left(\mathrm{P}\left\{M_{1}>x\right\}\right)$. Note that there exists a function $h(x) \rightarrow \infty$ such that $x-h(x) \rightarrow \infty$ and $\mathrm{P}\left\{X_{1}>h(x)\right\}=o\left(\mathrm{P}\left\{M_{1}>x\right\}\right)$. Now distinguish between the two cases $X_{1}>h(x)$ and $X_{1} \leq h(x)$, to obtain

$\mathrm{P}\left\{M_{1}>x, X_{1}+Y>x\right\} \leq \mathrm{P}\left\{X_{1} \geq h(x)\right\}+\mathrm{P}\left\{M_{1}>x\right\} \mathrm{P}\{Y>x-h(x)\}=o\left(\mathrm{P}\left\{M_{1}>x\right\}\right)$,

which implies the statement. 
Proof of Theorem 2. As in [16], we use the fact that $M \stackrel{\mathrm{D}}{=} \max \left\{M_{1}, X_{1}+\tilde{M}\right\}$, where $\tilde{M}$ is an independent copy of $M$ which is independent of $X_{1}$ and $M_{1}$. In addition, we use the following stochastic comparison argument. If $Y_{1}^{\downarrow}$ and $Y_{1}^{\uparrow}$ are two random variables independent of $\left(X_{1}, M_{1}\right)$, then $Y_{1}^{\downarrow} \geq_{\text {st }} M$ if and only if $Y_{1}^{\downarrow} \geq_{\text {st }} \max \left\{M_{1}, X_{1}+Y_{1}^{\downarrow}\right\}$. Similarly, $Y_{1}^{\uparrow} \leq_{\text {st }} M$ if and only if $Y_{1}^{\uparrow} \leq_{\text {st }} \max \left\{M_{1}, X_{1}+Y_{1}^{\uparrow}\right\}$.

To prove an asymptotic lower bound, define a sequence of random variables $Y_{n}^{\uparrow}, n \geq 1$, where $Y_{1}^{\uparrow}=M_{1}$ and $Y_{n+1}^{\uparrow}=\max \left\{M_{n+1}, X_{n+1}+Y_{n}^{\uparrow}\right\}, n \geq 1$. Using the above stochastic comparison argument $n$ times, we find that $\mathrm{P}\{M>x\} \geq \mathrm{P}\left\{Y_{n}^{\uparrow}>x\right\}$ for every $n$. By repeated application of Lemma 1, we have

$$
\mathrm{P}\left\{Y_{n}^{\uparrow}>x\right\} \sim \frac{1-\mathrm{E}\left\{\mathrm{e}^{\nu X_{1}}\right\}^{n}}{1-\mathrm{E}\left\{\mathrm{e}^{v X_{1}}\right\}} \mathrm{P}\left\{M_{1}>x\right\} .
$$

Consequently,

$$
\liminf _{x \rightarrow \infty} \frac{\mathrm{P}\{M>x\}}{\mathrm{P}\left\{M_{1}>x\right\}} \geq \frac{1-\mathrm{E}\left\{\mathrm{e}^{\nu X_{1}}\right\}^{n}}{1-\mathrm{E}\left\{\mathrm{e}^{v X_{1}}\right\}},
$$

which implies the desired asymptotic lower bound by letting $n \rightarrow \infty$.

To prove an asymptotic upper bound, we again use an idea from [16]. Let $C>1 /(1-$ $\left.\mathrm{E}\left\{\mathrm{e}^{v X_{1}}\right\}\right)$ and let $Y$ be a random variable independent of $\left(M_{1}, X_{1}\right)$ and such that $\mathrm{P}\{Y>x\} \sim$ $C \mathrm{P}\left\{M_{1}>x\right\}$. By Lemma 1 , there exists an $x_{0}<\infty$ such that

$$
\mathrm{P}\{Y>x\} \geq \mathrm{P}\left\{\max \left\{M_{1}, X_{1}+Y\right\}>x\right\} \text { for } x \geq x_{0} .
$$

Define $Y_{1}^{\downarrow}$ as an independent random variable such that $\mathrm{P}\left\{Y_{1}^{\downarrow}>x\right\}=\mathrm{P}\{Y>x\} / \mathrm{P}\left\{Y>x_{0}\right\}$. As in Lemma 3 of [16], it is easy to verify that $Y_{1}^{\downarrow} \geq_{\text {st }} \max \left\{M_{1}, X_{1}+Y_{1}^{\downarrow}\right\}$. Now define $Y_{n+1}^{\downarrow}=\max \left\{M_{n+1}, X_{n+1}+Y_{n}^{\downarrow}\right\}$ for $n \geq 1$. The remainder of the proof is similar to the proof of the lower bound. Applying the comparison argument, we see that $\mathrm{P}\{M>x\} \leq \mathrm{P}\left\{Y_{n}^{\downarrow}>x\right\}$. Set $C_{0}=C / \mathrm{P}\left\{Y>x_{0}\right\}$. By Lemma 2 and induction, we have

$$
\mathrm{P}\left\{Y_{n}^{\downarrow}>x\right\} \sim\left(\frac{1-\mathrm{E}\left\{\mathrm{e}^{v X_{1}}\right\}^{n-1}}{1-\mathrm{E}\left\{\mathrm{e}^{\nu X_{1}}\right\}}+C_{0} \mathrm{E}\left\{\mathrm{e}^{\nu X_{1}}\right\}^{n-1}\right) \mathrm{P}\left\{M_{1}>x\right\} .
$$

Combining these results, we obtain

$$
\lim _{n \rightarrow \infty} \limsup _{x \rightarrow \infty} \frac{\mathrm{P}\{M>x\}}{\mathrm{P}\left\{M_{1}>x\right\}} \leq \frac{1}{1-\mathrm{E}\left\{\mathrm{e}^{v X_{1}}\right\}},
$$

which completes the proof of the upper bound.

\subsection{The intermediate case}

We now consider the case in which $X_{1}$ satisfies

$$
\mathrm{P}\left\{X_{1}>x+y\right\} \sim \mathrm{e}^{-\alpha y} \mathrm{P}\left\{X_{1}>x\right\}
$$

as $x \rightarrow \infty$, for every fixed $y$, and

$$
\mathrm{P}\left\{X_{1}+X_{2}>x\right\} \sim 2 \mathrm{E}\left\{\mathrm{e}^{\alpha X_{1}}\right\} \mathrm{P}\left\{X_{1}>x\right\} .
$$

This is equivalent to the condition that $X_{1}^{+} \in \delta(\alpha)$. The case $\alpha=0$ was treated in Subsection 2.1, so we assume that $\alpha>0$. Another assumption we make is that

$$
\mathrm{E}\left\{\mathrm{e}^{\alpha X_{1}}\right\}<1,
$$


which implies that Cramér's condition is not satisfied. Finally, we specify the tail behavior of $M_{1}$. The case in which $M_{1}$ has a heavier tail than $X_{1}$ was covered in the previous subsection. Motivated by this, we assume that

$$
\lim _{x \rightarrow \infty} \frac{\mathrm{P}\left\{M_{1}>x\right\}}{\mathrm{P}\left\{X_{1}>x\right\}}<\infty
$$

(we allow the limit to equal 0). Furthermore, we assume that there exists a bounded function $f$ such that

$$
\lim _{x \rightarrow \infty} \frac{\mathrm{P}\left\{M_{1}>x, X_{1} \leq x-a\right\}}{\mathrm{P}\left\{X_{1}>x\right\}}=f(a)
$$

for all real values of $a$. This covers the case in which $M_{1}$ and $X_{1}$ are independent (in which $f$ is constant) and the random walk case (in which $M_{1}=0$, meaning that $f(a)=0$ ). An example in the regenerative setting can be found in Section 3.

We are now ready to state and prove our third main result. The method we use is similar to the one employed in the previous subsection, and also provides a new proof of the random walk case. As a preliminary result, we need the following lemma, which plays the same role as Lemma 1 in the previous subsection.

Lemma 2. Suppose that (5)-(9) are satisfied and that $Y$ is independent of $\left(M_{1}, X_{1}\right)$ with $\mathrm{P}\{Y>x\} \sim C_{Y} \mathrm{P}\left\{X_{1}>x\right\}$. Then

$$
\mathrm{P}\left\{\max \left\{M_{1}, X_{1}+Y\right\}>x\right\} \sim\left(\mathrm{E}\{f(Y)\}+\mathrm{E}\left\{\mathrm{e}^{\alpha Y}\right\}+C_{Y} \mathrm{E}\left\{\mathrm{e}^{\alpha X_{1}}\right\}\right) \mathrm{P}\left\{X_{1}>x\right\} .
$$

Proof. Write

$$
\mathrm{P}\left\{\max \left\{M_{1}, X_{1}+Y\right\}>x\right\}=\mathrm{P}\left\{X_{1}+Y>x\right\}+\mathrm{P}\left\{M_{1}>x, X_{1}+Y \leq x\right\} .
$$

The first term is asymptotically equivalent to $\left(\mathrm{E}\left\{\mathrm{e}^{\alpha Y}\right\}+C_{Y} \mathrm{E}\left\{\mathrm{e}^{\alpha X_{1}}\right\}\right) \mathrm{P}\left\{X_{1}>x\right\}$, using a result of [7]. To estimate the second term, note that (8) allows us to apply the bounded convergence theorem, which yields

$$
\lim _{x \rightarrow \infty} \frac{\mathrm{P}\left\{M_{1}>x, X_{1}+Y \leq x\right\}}{\mathrm{P}\left\{X_{1}>x\right\}}=\lim _{x \rightarrow \infty} \frac{\int \mathrm{P}\left\{M_{1}>x, X_{1} \leq x-y\right\} \mathrm{dP}\{Y \leq y\}}{\mathrm{P}\left\{X_{1}>x\right\}}=\mathrm{E}\{f(Y)\},
$$

which completes the proof of the lemma.

Theorem 3. Suppose that (5)-(9) are satisfied. Then

$$
\mathrm{P}\{M>x\} \sim \frac{\mathrm{E}\left\{\mathrm{e}^{\alpha M}\right\}+\mathrm{E}\{f(M)\}}{1-\mathrm{E}\left\{\mathrm{e}^{\alpha X_{1}}\right\}} \mathrm{P}\left\{X_{1}>x\right\} .
$$

Proof. As in the proof of Theorem 2, we make use of stochastic ordering arguments. The sequence $\left(Y_{n}^{\uparrow}\right)$ is defined as before: $Y_{1}^{\uparrow}=M_{1}$ and $Y_{n+1}^{\uparrow}=\max \left\{M_{n+1}, X_{n+1}+Y_{n}^{\uparrow}\right\}, n \geq 1$. Using the stochastic comparison argument $n$ times, we find that $\mathrm{P}\{M>x\} \geq \mathrm{P}\left\{Y_{n}^{\uparrow}>x\right\}$, which holds for every $n$. By repeated application of Lemma 2, we have

$$
\mathrm{P}\left\{Y_{n}^{\uparrow}>x\right\} \sim C_{Y_{n}^{\uparrow}} \mathrm{P}\left\{X_{1}>x\right\}, \quad n \geq 2,
$$

where $C_{Y_{1}^{\uparrow}}$ is the limit in (8) and

$$
C_{Y_{n+1}^{\uparrow}}=\mathrm{E}\left\{f\left(Y_{n}^{\uparrow}\right)\right\}+\mathrm{E}\left\{\mathrm{e}^{\alpha Y_{n}^{\uparrow}}\right\}+C_{Y_{n}^{\uparrow}} \mathrm{E}\left\{\mathrm{e}^{\alpha X_{1}}\right\}, \quad n \geq 1 .
$$


Consequently,

$$
\liminf _{x \rightarrow \infty} \frac{\mathrm{P}\{M>x\}}{\mathrm{P}\left\{X_{1}>x\right\}} \geq C_{Y_{n}^{\uparrow}}, \quad n \geq 1 .
$$

Since $Y_{n}^{\uparrow} \leq_{\mathrm{st}} Y_{n+1}^{\uparrow} \leq_{\mathrm{st}} M$ and $Y_{n}^{\uparrow} \stackrel{\mathrm{D}}{\rightarrow} M$, by bounded and monotone convergence we find that $C_{Y_{n}^{\uparrow}} \rightarrow C_{M}$, where $C_{M}$ satisfies

$$
C_{M}=\mathrm{E}\{f(M)\}+\mathrm{E}\left\{\mathrm{e}^{\alpha M}\right\}+C_{M} \mathrm{E}\left\{\mathrm{e}^{\alpha X_{1}}\right\} .
$$

This completes the proof of the asymptotic lower bound.

To obtain an upper bound, we need to construct a random variable $Y_{1}^{\downarrow}$ with the property that $Y_{1}^{\downarrow} \geq_{\text {st }} \max \left\{M_{1}, X_{1}+Y_{1}^{\downarrow}\right\}$. Let $X$ be an independent copy of $X_{1}$ and let $Y=(X+T) \times$ $\mathbf{1}(X \geq y)$, with $y$ and $T$ large constants. Note that $\mathrm{P}\{Y>x\} \sim \mathrm{e}^{\alpha T} \mathrm{P}\left\{X_{1}>x\right\}$ and $\mathrm{E}\left\{\mathrm{e}^{\alpha Y}\right\}=\mathrm{e}^{\alpha T} \mathrm{E}\left\{\mathrm{e}^{\alpha X_{1}} \mathbf{1}\left(X_{1} \geq y\right)\right\}$. From Lemma 2 we have

$$
\mathrm{P}\left\{\max \left\{M_{1}, X_{1}+Y\right\}>x\right\} \sim\left(\mathrm{E}\{f(Y)\}+\mathrm{E}\left\{\mathrm{e}^{\alpha Y}\right\}+\mathrm{e}^{\alpha T} \mathrm{E}\left\{\mathrm{e}^{\alpha X_{1}}\right\}\right) \mathrm{P}\left\{X_{1}>x\right\} .
$$

Using the facts that $\mathrm{E}\{f(Y)\}$ is bounded and $\mathrm{E}\left\{\mathrm{e}^{\alpha X_{1}}\right\}<1$, we can choose $T$ and $y$ such that the prefactor $\mathrm{E}\{f(Y)\}+\mathrm{E}\left\{\mathrm{e}^{\alpha Y}\right\}+\mathrm{e}^{\alpha T} \mathrm{E}\left\{\mathrm{e}^{\alpha X_{1}}\right\}$ is smaller than $\mathrm{e}^{\alpha T}$. Consequently, there exists a value $x_{0}$ such that $\mathrm{P}\{Y>x\} \geq \mathrm{P}\left\{\max \left\{M_{1}, X_{1}+Y\right\}>x\right\}$ for $x \geq x_{0}$.

Now define $Y_{1}^{\downarrow}$ as a random variable independent of $\left(M_{1}, X_{1}\right)$ and such that

$$
\mathrm{P}\left\{Y_{1}^{\downarrow}>x\right\}=\frac{\mathrm{P}\{Y>x\}}{\mathrm{P}\left\{Y>x_{0}\right\}}=\mathrm{P}\left\{Y>x \mid Y>x_{0}\right\} .
$$

As in Lemma 3 of [16], for $x \geq x_{0}$ we see that

$$
\begin{aligned}
\mathrm{P}\left\{\max \left\{M_{1}, X_{1}+Y_{1}^{\downarrow}\right\}>x\right\} & =\mathrm{P}\left\{\max \left\{M_{1}, X_{1}+Y\right\}>x \mid Y>x_{0}\right\} \\
& \leq \frac{\mathrm{P}\left\{\max \left\{M_{1}, X_{1}+Y\right\}>x\right\}}{\mathrm{P}\left\{Y>x_{0}\right\}} \\
& \leq \frac{\mathrm{P}\{Y>x\}}{\mathrm{P}\left\{Y>x_{0}\right\}} \\
& =\mathrm{P}\left\{Y_{1}^{\downarrow}>x\right\} .
\end{aligned}
$$

The inequality is trivial for $x<x_{0}$, so we see that $Y_{1}^{\downarrow} \geq_{\text {st }} \max \left\{X_{1}, M_{1}+Y_{1}^{\downarrow}\right\}$. The proof is now completed by defining $Y_{n}^{\downarrow}, n \geq 2$, as in the proof of Lemma 1 . Since $M \leq_{\text {st }} Y_{n}^{\downarrow}$ for any $n$, by applying Lemma 2 we obtain

$$
\limsup _{x \rightarrow \infty} \frac{\mathrm{P}\{M>x\}}{\mathrm{P}\left\{X_{1}>x\right\}} \leq C_{Y_{n}^{\downarrow}},
$$

with $C_{Y_{n}^{\uparrow}}$ defined in a way similar to that in the proof of the lower bound. Using the same arguments as in the proof of the lower bound, we find that $C_{Y_{n}^{\downarrow}} \rightarrow C_{M}$, which completes the proof.

\subsection{The Cramér case}

In this subsection we review the extension of the classical Cramér case from random walks to perturbed random walks and regenerative processes. This problem has also been considered in [25] and [1]. The result presented here is an extension of these two works, and it follows from Theorem 5.2 of [14]. Actually, there the equation $R \stackrel{\mathrm{D}}{=} \max \{A \tilde{R}, B\}$, which can easily be reduced to our equation for $M$ by taking logarithms, was considered. 
Theorem 4. (Theorem 5.2 of [14].) Assume that there exists a solution $\kappa>0$ to the equation $\mathrm{E}\left\{\mathrm{e}^{\kappa X_{1}}\right\}=1$ such that $m=\mathrm{E}\left\{X_{1} \mathrm{e}^{\kappa X_{1}}\right\}<\infty$. Assume, furthermore, that $X_{1}$ is nonlattice and that $\mathrm{E}\left\{\mathrm{e}^{\kappa M_{1}}\right\}<\infty$. Then

$$
\mathrm{P}\{M>x\} \sim K \mathrm{e}^{-\kappa x} \quad \text { with } \quad K=\frac{1}{\kappa m} \mathrm{E}\left\{\mathrm{e}^{\kappa M_{1}}-\mathrm{e}^{\kappa\left(\tilde{M}+X_{1}\right)} ; M_{1}>\tilde{M}+X_{1}\right\} .
$$

It is easy to see that $K$ is bounded from above by $\bar{K}=\mathrm{E}\left\{\mathrm{e}^{\kappa M_{1}}\right\} /(\kappa m)$. If $M_{1}$ is nonnegative then $K$ is bounded from below by the prefactor $C_{W}$ in the Cramér-Lundberg expansion $\mathrm{P}\{W>x\} \sim C_{W} \mathrm{e}^{-\kappa x}$, with $W=\sup _{n \geq 0} S_{n}$; see [2, Theorem XIII.5.3, p. 365].

\section{A fluid model}

To illustrate the general theory developed in the previous section, we now investigate a simple example. Let $J(t), t \geq 0$, be an alternating renewal (0-1) process with generic onperiod $T_{\text {on }}$ and generic off-period $T_{\text {off }}$, i.e. $T_{\text {on }}$ is the period during which $J(s)=1$ and $T_{\text {off }}$ is the period during which $J(s)=0$. Let $Y(t)=r \int_{0}^{t} J(s) \mathrm{d} s, t \geq 0$, be the associated integrated on-off process. The constant $r>0$ is called the on rate. Assume that $J(t)$ is such that an on-period starts at time 0 . Let the sequence $\left(T_{\mathrm{on}, i}, T_{\mathrm{off}, i}\right), i \geq 1$, representing on-times and offtimes, be independent and identically distributed with $\left(T_{\text {on, } 1}, T_{\text {off }, 1}\right) \stackrel{\text { D }}{=}\left(T_{\text {on }}, T_{\text {off }}\right)$. We allow $T_{\mathrm{on}}$ and $T_{\mathrm{off}}$ to be dependent and assume that $T_{\mathrm{on}}+T_{\mathrm{off}}$ has finite mean. Assume further that $\mathrm{E}\{J(t)\} \rightarrow \rho \in(0, c)$ for some constant $c>0$ called the drain rate. Under these conditions, the process $S(t)=Y(t)-c t, t \geq 0$, is almost surely convergent to $-\infty$. The renewal epochs for the process $S(t), t \geq 0$, are given by $T_{i}=\sum_{k=1}^{i}\left(T_{\mathrm{on}, k}+T_{\mathrm{off}, k}\right), i \geq 0$. In this setting, the distribution of $M$ can be viewed as the Palm stationary distribution of the amount of fluid in a buffer fed by an on-off source. This is a simple and well-known model (see, e.g. [17] and [19]), and as such it provides simple applications of the theory developed in the previous section. In the setting of that section, we have $X_{1}=(r-c) T_{\text {on }}-c T_{\text {off }}, M_{1}=(r-c)^{+} T_{\text {on }}$, and, hence, $M_{1}^{*}=M_{1}$.

\subsection{An application of Theorems 1,2 , and 4}

Assume that $r>c$ and that $T_{\text {on }}$ and $T_{\text {off }}$ are dependent in the following way: let $E_{0}, E_{1}$, and $E_{2}$ be independent random variables with finite means, and suppose that $T_{\text {on }}=E_{0}+E_{1}$ and $T_{\text {off }}=E_{0}+E_{2}$. In this case,

$$
M_{1}=(r-c) E_{0}+(r-c) E_{1}, \quad X_{1}=(r-c) E_{1}+(r-2 c) E_{0}-c E_{2} .
$$

Moreover, $\mathrm{E}\left\{X_{1}\right\}$ is assumed to be strictly negative. We now focus on two different scenarios.

- Assume that $\mathrm{P}\left\{E_{0}>x\right\}$ is long tailed, that $\int_{x}^{\infty} \mathrm{P}\left\{E_{0}>u\right\} \mathrm{d} u$ is subexponential, and that $E_{1}$ has a finite moment generating function in a neighborhood of the origin. Then $\mathrm{P}\left\{M_{1}>x\right\} \sim \mathrm{P}\left\{(r-c) E_{0}>x\right\}$, implying that the conditions of Theorem 1 are satisfied. The property $\mathrm{P}\left\{M_{1}>x\right\} \sim \mathrm{P}\left\{X_{1}>x\right\}$ is clearly not satisfied. If $E_{0}$ has a lognormal or heavy-tailed Weibull distribution, it can actually be shown that

$$
\mathrm{P}\left\{X_{1}>x\right\} \sim o\left(\mathrm{P}\left\{M_{1}>x\right\}\right)
$$

we omit the details. 
- Assume that $\mathrm{P}\left\{E_{0}>x\right\} \sim c_{0} \mathrm{e}^{-v_{0} x}$ and that $\mathrm{E}\left\{\mathrm{e}^{\nu_{0} E_{1}}\right\}<\infty$. Then

$$
\mathrm{P}\left\{M_{1}>x\right\} \sim c_{0} \mathrm{E}\left\{\mathrm{e}^{v_{0} E_{1}}\right\} \exp \left\{-\frac{v_{0}}{r-c} x\right\}=: \bar{c}_{0} \mathrm{e}^{-\bar{v}_{0} x} .
$$

Furthermore, we have $\mathrm{E}\left\{\mathrm{e}^{\overline{\mathrm{v}}_{0} X_{1}}\right\}<\infty$. If this quantity is strictly larger than 1 , then we are in the Cramér case covered by Theorem 4 . If the quantity is strictly less than 1 , then the tail behavior of $\mathrm{P}\{M>x\}$ follows from Theorem 2 .

\subsection{An application of Theorem 3}

Assume that $r>c$, that $T_{\mathrm{on}}$ and $T_{\mathrm{off}}$ are independent, and that $T_{\mathrm{on}}$ is in $\delta(\alpha), \alpha>0$. Then $M_{1}$ is in $\delta(\alpha)$ as well, and $\mathrm{P}\left\{X_{1}>x\right\} \sim \mathrm{E}\left\{\mathrm{e}^{-\alpha c T_{\text {off }}}\right\} \mathrm{P}\left\{M_{1}>x\right\}$. Since the class $\delta(\alpha)$ is closed under tail equivalence, we see that $X_{1}^{+} \in \delta(\alpha)$, which implies (5) and (6). Assume further that (7) holds. To apply Theorem 3, it remains to verify condition (9). To do so, we write

$$
\begin{aligned}
\mathrm{P}\left\{M_{1}>x, X_{1} \leq x-a\right\} & =\mathrm{P}\left\{M_{1}>x, M_{1}-c T_{\text {off }} \leq x-a\right\} \\
& =\int_{0}^{\infty} \mathrm{P}\left\{x<M_{1} \leq x+u\right\} \mathrm{dP}\left\{c T_{\text {off }}-a \leq u\right\} \\
& \sim \mathrm{P}\left\{M_{1}>x\right\} \int_{0}^{\infty}\left(1-\mathrm{e}^{-\alpha u}\right) \mathrm{d} \mathrm{P}\left\{c T_{\text {off }}-a \leq u\right\} \\
& =\mathrm{P}\left\{M_{1}>x\right\}\left(\mathrm{P}\left\{c T_{\text {off }} \geq a\right\}-\mathrm{E}\left\{\mathrm{e}^{-\alpha\left(c T_{\text {off }}-a\right)} \mathbf{1}\left(c T_{\text {off }} \geq a\right)\right\}\right),
\end{aligned}
$$

where we have applied the property (5) for $M_{1}$ in the third step. The tail behavior of $M$ now follows from Theorem 3, with $f(a)=\mathrm{E}\left\{\left(1-\mathrm{e}^{-\alpha\left(c T_{\text {off }}-a\right)}\right) \mathbf{1}\left(c T_{\text {off }} \geq a\right)\right\} / \mathrm{E}\left\{\mathrm{e}^{-\alpha c T_{\text {off }}}\right\}$.

\subsection{A fluid model with noise}

In this subsection we provide another application of Theorem 1. Let $r=c=1$ and let $S(t)=Y(t)+W(t)-t$ with $W(t), t \geq 0$, an independent standard Wiener process. The process $S(t), t \geq 0$, can be interpreted as the net input process of an on-off fluid model perturbed by Brownian motion, with the additional feature that the on rate equals the drain rate (both are equal to 1).

The sequence $T_{n}, n \geq 1$, representing the starting points of on-periods, is again a renewal sequence of the process $S(t), t \geq 0$. It is clear that

$$
\left(M_{1}, X_{1}\right) \stackrel{\mathrm{D}}{=}\left(\sup _{0<t \leq T_{\text {on }}+T_{\text {off }}}\left[W(t)-\left(t-T_{\text {on }}\right)^{+}\right], W\left(T_{\text {on }}+T_{\text {off }}\right)-T_{\text {off }}\right) .
$$

Assume that $\mathrm{P}\left\{T_{\text {on }}>x\right\}$ is regularly varying of index $-v, v>1$. We first state some preliminary results. Combining Theorem 2.1 and Proposition 2.1(i) of [8] we obtain

$$
\mathrm{P}\left\{\sup _{0<t<T_{\text {on }}} W(t)>x\right\} \sim \frac{1}{\sqrt{\pi}} 2^{v+1} \Gamma\left(v+\frac{1}{2}\right) \mathrm{P}\left\{T_{\text {on }}>x^{2}\right\} .
$$

Using a famous result of P. Lévy [23, p. 240], we have that

$$
\mathrm{P}\left\{\sup _{0<t<T_{\text {on }}} W(t)>x\right\}=\mathrm{P}\left\{\left|W\left(T_{\text {on }}\right)\right|>x\right\} .
$$

By using the symmetry around 0 of the standard normal distribution, we conclude that

$$
\mathrm{P}\left\{W\left(T_{\text {on }}\right)>x\right\}=\frac{1}{2} \mathrm{P}\left\{\left|W\left(T_{\text {on }}\right)\right|>x\right\} \sim \frac{1}{\sqrt{\pi}} 2^{v} \Gamma\left(v+\frac{1}{2}\right) \mathrm{P}\left\{T_{\text {on }}>x^{2}\right\} .
$$


In particular, the right tails of the distributions of $W\left(T_{\mathrm{on}}\right)$ and $\sup _{0<t<T_{\mathrm{on}}} W(t)$ are regularly varying with index $-2 v$. This is an important ingredient in the proof of the following result.

Lemma 3. In the setting of this subsection, we have

$$
\mathrm{P}\left\{M_{1}^{*}>x\right\}=\mathrm{P}\left\{M_{1}>x\right\} \sim \mathrm{P}\left\{\sup _{0<t<T_{\text {on }}} W(t)>x\right\}, \quad \mathrm{P}\left\{X_{1}>x\right\} \sim \mathrm{P}\left\{W\left(T_{\text {on }}\right)>x\right\} .
$$

Proof. First we prove the assertion for $M_{1}$. From the definition of $M_{1}$ it immediately follows that

$$
\liminf _{x \rightarrow \infty} \frac{\mathrm{P}\left\{M_{1}>x\right\}}{\mathrm{P}\left\{\sup _{0<t<T_{\text {on }}} W(t)>x\right\}} \geq 1 .
$$

To prove an asymptotic upper bound, we use $T_{\text {off }}<\infty$ to obtain

$$
\begin{aligned}
& M_{1} \leq \sup _{t>0}\left[W(t)-\left(t-T_{\text {on }}\right)^{+}\right] \\
& =\max \left\{\sup _{0<t<T_{\text {on }}}[W(t)], W\left(T_{\text {on }}\right)+\sup _{t>T_{\text {on }}}\left[W(t)-W\left(T_{\text {on }}\right)-\left(t-T_{\text {on }}\right)\right]\right\} \\
& \leq \sup _{0<t<T_{\mathrm{on}}}[W(t)]+\sup _{t>T_{\mathrm{on}}}\left[W(t)-W\left(T_{\mathrm{on}}\right)-\left(t-T_{\mathrm{on}}\right)\right] .
\end{aligned}
$$

Since the increments of the Wiener process are independent, it is clear that the two terms in the last line are independent. Furthermore, the random variable $\sup _{t>T_{\mathrm{on}}}\left[W(t)-W\left(T_{\mathrm{on}}\right)-\left(t-T_{\mathrm{on}}\right)\right]$ has an exponential distribution with mean $\frac{1}{2}$; see, e.g. [2, Example IX.3.5, p. 258]. Moreover, the right tail of $\sup _{0<t<T_{\mathrm{on}}} W(t)$ is regularly varying with index $-2 v$, which leads us to

$$
\mathrm{P}\left\{\sup _{0<t<T_{\mathrm{on}}}[W(t)]+\sup _{t>T_{\mathrm{on}}}\left[W(t)-W\left(T_{\mathrm{on}}\right)-\left(t-T_{\mathrm{on}}\right)\right]>x\right\} \sim \mathrm{P}\left\{\sup _{0<t<T_{\mathrm{on}}} W(t)>x\right\} .
$$

Consequently,

$$
\limsup _{x \rightarrow \infty} \frac{\mathrm{P}\left\{M_{1}>x\right\}}{\mathrm{P}\left\{\sup _{0<t<T_{\text {on }}} W(t)>x\right\}} \leq 1,
$$

which implies our first assertion.

To prove the second assertion, let $\tilde{W}$ be another standard Wiener process, independent of $W, T_{\text {on }}$, and $T_{\text {off }}$. We first prove an asymptotic lower bound. Note that

$$
X_{1} \stackrel{\mathrm{D}}{=} W\left(T_{\text {on }}\right)+\tilde{W}\left(T_{\text {off }}\right)-T_{\text {off }} .
$$

Consequently, for any $y>0$,

$$
\mathrm{P}\left\{X_{1}>x\right\} \geq \mathrm{P}\left\{W\left(T_{\text {on }}\right)>x+y\right\} \mathrm{P}\left\{\tilde{W}\left(T_{\text {off }}\right)-T_{\text {off }}>-y\right\} .
$$

The random variable $W\left(T_{\text {on }}\right)$ has a right tail which is regularly varying with index $-2 v$. In particular, its right tail is long tailed. This implies that

$$
\liminf _{x \rightarrow \infty} \frac{\mathrm{P}\left\{X_{1}>x\right\}}{\mathrm{P}\left\{W\left(T_{\text {on }}\right)>x\right\}} \geq \mathrm{P}\left\{\tilde{W}\left(T_{\text {off }}\right)-T_{\text {off }}>-y\right\} .
$$

The asymptotic lower bound now follows by letting $y \rightarrow \infty$. We now turn to the upper bound. Since

$$
\mathrm{P}\left\{\tilde{W}\left(T_{\text {off }}\right)-T_{\text {off }}>x\right\} \leq \mathrm{P}\left\{\sup _{t>0}[\tilde{W}(t)-t]>x\right\}=\mathrm{e}^{-2 x},
$$

the upper bound follows by an argument similar to that made for $M_{1}$. 
Putting everything together, it follows that $\mathrm{P}\left\{M_{1}>x\right\} \sim 2 \mathrm{P}\left\{X_{1}>x\right\}$, and both tails are regularly varying with index $-2 v$. Using Theorem 1 , this yields

$$
\mathrm{P}\{M>x\} \sim \frac{1}{\mathrm{E}\left\{T_{\text {off }}\right\}} \frac{1}{\sqrt{\pi}} 2^{v+1} \Gamma\left(v+\frac{1}{2}\right) \int_{x}^{\infty} \mathrm{P}\left\{T_{\text {on }}>u^{2}\right\} \mathrm{d} u .
$$

To conclude, it is interesting to note that the tail asymptotics of $M$ in the zero-delayed case discussed here are regularly varying with index $1-2 v$. This differs significantly from the tail behavior in the delayed (stationary) case, which is regularly varying with index $2-2 v$; see Theorem 4.1 of [29].

\section{A model from insurance risk}

Consider the regenerative process $S(t), t \geq 0$, given by

$$
S(t)=\sum_{i=1}^{N(t)} U_{i}-t, \quad t \geq 0,
$$

where $N(t), t \geq 0$, is a Cox process with an underlying regenerative process $R(t), t \geq 0$, with renewal epochs $T_{i}, i \geq 1$. That is, there exists a nonnegative measurable function $\lambda: \mathbb{R} \rightarrow$ $\mathbb{R}_{+} \cup\{0\}$ with the following property: for a realization $r(t), t \geq 0$, of the process $R(t), t \geq 0$, the process $N(t), t \geq 0$, has the same law as an inhomogeneous Poisson process with intensity $\bar{\lambda}(t)=\lambda(r(t))$ at time $t \geq 0$. Detailed discussions of Cox processes and their impact on risk theory can be found in [15], [24], [5], [25], and [3]. The claim sizes $U_{i}, i \geq 1$, are independent, identically distributed random variables independent of the process $N(t), t \geq 0$, with a common nonlattice distribution function $F_{U}$. Let $x$ be the initial reserve and assume that $S(t) \rightarrow-\infty$ almost surely, as $t \rightarrow \infty$. The process $x-S(t), t \geq 0$, is known as the surplus process, and we say that ruin occurs if this process hits 0 . The infinite-horizon ruin probability is then given by

$$
\psi(x)=\mathrm{P}\{M>x\} .
$$

\subsection{Applications of Theorem 4}

We first focus on the Cramér case covered by Theorem 4. That is, we assume that there exists a constant $\kappa>0$ such that

$$
\mathrm{E}\left\{\mathrm{e}^{\kappa S\left(T_{1}\right)}\right\}=\mathrm{E} \exp \left\{\int_{0}^{T_{1}} \lambda(R(s)) \mathrm{d} s\left(\hat{m}_{U}(\kappa)-1\right)-\kappa T_{1}\right\}=1,
$$

where $\hat{m}_{U}(\theta)=\mathrm{E}\left\{\mathrm{e}^{\theta U}\right\}$. In risk theory, $\kappa$ is called the adjustment coefficient. In addition, we assume that

$$
m=\mathrm{E}\left\{\hat{m}_{U}^{\prime}(\kappa) \int_{0}^{T_{1}} \lambda(R(s)) \mathrm{d} s-T_{1}\right\} \exp \left\{\int_{0}^{T_{1}} \lambda(R(s)) \mathrm{d} s\left(\hat{m}_{U}(\kappa)-1\right)-\kappa T_{1}\right\}<\infty
$$

and that

$$
\mathrm{E}\left\{\exp \left\{\int_{0}^{T_{1}} \lambda(R(s)) \mathrm{d} s\left(\hat{m}_{U}(\kappa)-1\right)\right\}\right\}<\infty .
$$

From Theorem 4 we derive the exponential asymptotics

$$
\psi(x) \sim K \mathrm{e}^{-\kappa x}
$$


Note that the constant $K$ can be bounded from above by

$$
\bar{K}=\frac{1}{\kappa m} \mathrm{E}\left\{\exp \left\{\kappa \sum_{i=1}^{N\left(T_{1}\right)} U_{i}\right\}\right\}=\frac{1}{\kappa m} \mathrm{E}\left\{\exp \left\{\int_{0}^{T_{1}} \lambda(X(s)) \mathrm{d} s\left(\hat{m}_{U}(\kappa)-1\right)\right\}\right\} .
$$

An important special case is the Björk-Grandell model, where $\lambda(x)=x$ and $R(s)=L_{i}$ for $\sum_{j=1}^{i-1} \sigma_{j} \leq s<\sum_{j=1}^{i} \sigma_{j}$, with $\left(L_{i}, \sigma_{i}\right), i \geq 1$, an independent, identically distributed sequence of vectors with positive components. In this particular case, the adjustment coefficient $\kappa>0$ is a solution to the equation

$$
\mathrm{E}\left\{\exp \left\{\sigma L\left(\hat{m}_{U}(\kappa)-1\right)-\kappa \sigma\right\}\right\}=1,
$$

where $(\sigma, L)$ are generic $\left(\sigma_{i}, L_{i}\right)$. The upper bound of $K$ satisfies

$$
\bar{K}=(1 / \kappa m) \mathrm{E}\left\{\exp \left\{\sigma L\left(\hat{m}_{U}(\kappa)-1\right)\right\}\right\}
$$

with

$$
m=\mathrm{E}\left\{\left(\hat{m}_{U}^{\prime}(\kappa) \sigma L-\sigma\right) \exp \left\{\sigma L\left(\hat{m}_{U}(\kappa)-1\right)-\kappa \sigma\right\}\right\} .
$$

Existence of the exponential asymptotics was first established in [25]. A different upper bound for $K$ was derived in Theorem 12.5.3 of [24].

We now discuss an example of a Cox process where the intensity process is described by a diffusion process. A motivating example comes from vehicle insurance, where the intensity of the claim arrivals may depend on the density of vehicles insured. The latter can randomly change in time due to the variability of the number of inhabitants, or market share within an area. A functional of a diffusion process seems flexible enough to take the stochastic variability of the intensity process into account; we refer the reader to [26] for further discussion and motivation. We can use the exponential asymptotics given by (10) to obtain an exponential approximation of the ruin probability in this model. We consider the example of an Ornstein-Uhlenbeck process $R(t), t \geq 0$, which starts at 0 and has generator

$$
(\mathcal{A} f)(x)=\frac{1}{2} \frac{\mathrm{d}^{2}}{\mathrm{~d} x^{2}} f(x)-b x \frac{\mathrm{d}}{\mathrm{d} x} f(x),
$$

for some $b>0$. The domain of this generator contains all functions $f$ in $\mathcal{C}^{2}(\mathbb{R})$. In addition, we assume that $\lambda(x)=x^{2}+k$ for $k \geq 0$. In [22] it was shown that if $\mathrm{E}\{U\}<2 b /(1+2 b k)$ then $S(t)$ tends to $-\infty$ almost surely as $t \rightarrow \infty$; the inequality $\mathrm{E}\{U\}<2 b /(1+2 b k)$ will be assumed to hold from now on. Note that $R(t), t \geq 0$, is a regenerative process with regeneration epochs $T_{n+1}=\inf \left\{t \geq I_{n}: R(t)=0\right\}$, where $I_{n}=\inf \left\{t \geq T_{n}:|R(t)|=1\right\}, n \geq 0$.

Let $\eta>0$. Under $Q$, the twisted law of $R$ with respect to the martingale

$$
M(t)=\exp \left\{-\frac{\eta^{2}-b^{2}}{2} \int_{0}^{t} R^{2}(s) \mathrm{d} s-\frac{\eta-b}{2}\left(R^{2}(t)-R^{2}(0)-t\right)\right\}, \quad t \geq 0,
$$

we obtain an Ornstein-Uhlenbeck process with parameter $\eta$ (see [21]). Let

$$
Z=\int_{0}^{T_{1}} \lambda(R(s)) \mathrm{d} s=\int_{0}^{T_{1}}\left(R^{2}(s)+k\right) \mathrm{d} s
$$


and $\xi=\hat{m}_{U}(\kappa)-1$. Applying the twisted law with $\eta=\sqrt{b^{2}-2 \xi}$, we obtain the following equation for the adjustment coefficient $\kappa: \mathrm{E}_{Q}\left\{\mathrm{e}^{-(\hat{\eta}+\kappa) T_{1}}\right\}=1$, where $\hat{\eta}=-(b-\eta) / 2-\xi k$. Hence, $\hat{\eta}+\kappa=0$ and $\kappa$ solves the equation

$$
\kappa=\frac{b-\sqrt{b^{2}-2\left(\hat{m}_{U}(\kappa)-1\right)}}{2}+\left(\hat{m}_{U}(\kappa)-1\right) k .
$$

If the claims are exponentially distributed with mean $\varphi$ and $k=0$ then, for $\varphi<2 b$, we have

$$
\kappa=\frac{b \varphi+1}{2 \varphi}\left(1-\sqrt{1-\frac{2 \varphi(2 b-\varphi)}{(b \varphi+1)^{2}}}\right) .
$$

To the best of the author's knowledge, this is the first exact expression for the adjustment coefficient in a Cox model driven by a diffusion process. In addition, we can obtain an explicit expression for $\bar{K}$, the upper bound of $K$. Note that $T_{1}$ is the sum of the exit time from interval $[-1,1]$ and the (independent) first passage time into the negative half-line for the OrnsteinUhlenbeck process starting from 1 . Write $D_{-\zeta}$ for the parabolic cylinder function and set

$$
S(\zeta, x, y)=\frac{\Gamma(\zeta)}{\pi} \mathrm{e}^{\left(x^{2}+y^{2}\right) / 4}\left(D_{-\zeta}(-x) D_{-\zeta}(y)-D_{-\zeta}(x) D_{-\zeta}(-y)\right) .
$$

Using arguments similar to those in [22] and results from [6, p. 429, p. 434], for $\hat{\eta} / \eta>-2$ we obtain

$$
\bar{K}=\frac{1}{\kappa m} \mathrm{E}\left\{\mathrm{e}^{\xi Z}\right\}=\frac{1}{\kappa m} \mathrm{E}_{Q}\left\{\mathrm{e}^{-\hat{\eta} T_{1}}\right\}=\frac{1}{\kappa m} H(\hat{\eta}),
$$

where

$$
H(s)=\frac{S(s / \eta, \sqrt{2 \eta}, 0)+S(s / \eta, 0,-\sqrt{2 \eta})}{S(s / \eta, \sqrt{2 \eta},-\sqrt{2 \eta})} \frac{\mathrm{e}^{\eta / 2} D_{-s / \eta}(\sqrt{2 \eta})}{D_{-s / \eta}(0)}
$$

for $m=(\partial / \partial \kappa) H(\hat{\eta}+\kappa)$.

\subsection{An application of Theorem 2}

Consider the Björk-Grandell model with $\mathrm{P}\left\{L=\lambda_{i}\right\}=p_{i}>0, i=0,1, \ldots, d$. We assume that $\lambda_{0}>\lambda_{1}>\cdots>\lambda_{d}>0$ and that $\lambda_{0} \mathrm{E}\{U\}<1$. Moreover, let

$$
\mathrm{P}\left\{\sigma_{0}>x\right\}:=\mathrm{P}\left\{\sigma>x \mid L=\lambda_{0}\right\} \sim x^{-\alpha_{0}} l_{0}(x)
$$

for a slowly varying function $l_{0}$, and let there exist a solution $v_{0}>0$ to the equation $\phi_{0}(\theta)=$ $\lambda_{0}\left(\hat{m}_{U}(\theta)-1\right)-\theta=0$. We assume that $\hat{m}_{U}(\theta)$ is finite in a neighborhood of $v_{0}$. Also define $\phi_{i}(\theta)=\lambda_{i}\left(\hat{m}_{U}(\theta)-1\right)-\theta$. Writing $\tau(x)=\inf \{t \geq 0: S(t)>x\}$ for the ruin time, we find that

$$
\mathrm{P}\left\{M_{1}>x\right\}=\mathrm{P}\{\tau(x)<\sigma\}=\sum_{i=0}^{d} \mathrm{P}\left\{\tau(x)<\sigma \mid L=\lambda_{i}\right\} p_{i} .
$$

Our main goal is to determine the tail behavior of $M_{1}$, and to verify that the conditions of Theorem 2 are satisfied.

Proposition 1. In the setting of this subsection, we have

$$
\mathrm{P}\left\{M_{1}>x\right\} \sim p_{0} \mathrm{P}\left\{\sigma_{0}>\frac{x}{\phi_{0}^{\prime}\left(v_{0}\right)}\right\} \frac{-\phi_{0}^{\prime}(0)}{\phi_{0}^{\prime}\left(v_{0}\right)} \mathrm{e}^{-v_{0} x} .
$$


Proof. We first focus on the tail behavior of $\mathrm{P}\left\{M_{1}>x \mid L=\lambda_{0}\right\}$. A crucial observation is that, conditionally upon $L=\lambda_{0}$, the risk process evolves according to a standard compound Poisson process with rate $\lambda_{0}$ up to time $\sigma_{0}$. Let $\mathrm{P}_{0}\{\cdot\}$ be the probability measure under which the intensity equals $\lambda_{0}$ (we define $\mathrm{P}_{i}\{\cdot\}$ similarly). Then $\mathrm{P}\left\{M_{1}>x \mid L=\lambda_{0}\right\}=\mathrm{P}_{0}\left\{\tau(x)<\sigma_{0}\right\}$, meaning that $\sigma_{0}$ is independent of $(S(t))$ under $\mathrm{P}_{0}\{\cdot\}$. We now state two important results, which directly follow from results of [18]. Define $\hat{d}=1 / \phi_{0}^{\prime}\left(\nu_{0}\right)$ and let $\varepsilon>0$. Corollary 2.3 of [18] implies that

$$
\mathrm{P}_{0}\{\tau(x)<(\hat{d}+\varepsilon) x\} \sim \mathrm{P}_{0}\{\tau(x)<\infty\} \sim \frac{-\phi_{0}^{\prime}(0)}{\phi_{0}^{\prime}\left(v_{0}\right)} \mathrm{e}^{-v_{0} x} .
$$

Moreover, for every $\varepsilon \in(0, \hat{d})$, there exists a constant $\delta>0$ such that

$$
\mathrm{P}_{0}\{\tau(x)<(\hat{d}-\varepsilon) x\}=o\left(\mathrm{e}^{-\left(\nu_{0}+\delta\right) x}\right) .
$$

Therefore, from (11),

$$
\mathrm{P}_{0}\left\{\tau(x)<\sigma_{0}\right\} \geq \mathrm{P}_{0}\{\tau(x)<(\hat{d}+\varepsilon) x\} \mathrm{P}\left\{\sigma_{0}>(\hat{d}+\varepsilon) x\right\} .
$$

Combining this with the fact that $\mathrm{P}\left\{M_{1}>x\right\} \geq p_{0} \mathrm{P}_{0}\left\{\tau(x)<\sigma_{0}\right\}$, we obtain

$$
\liminf _{x \rightarrow \infty} \frac{\mathrm{P}\left\{M_{1}>x\right\}}{p_{0} \mathrm{P}\left\{\sigma_{0}>x / \phi_{0}^{\prime}\left(v_{0}\right)\right\}\left(-\phi_{0}^{\prime}(0) / \phi_{0}^{\prime}\left(\nu_{0}\right)\right) \mathrm{e}^{-v_{0} x}} \geq\left(\frac{\hat{d}}{\hat{d}+\varepsilon}\right)^{\alpha_{0}} .
$$

The proof of the lower bound is now completed by letting $\varepsilon \rightarrow 0$.

For the upper bound, observe that

$$
\mathrm{P}\left\{M_{1}>x\right\} \leq p_{0} \mathrm{P}_{0}\left\{\tau(x)<\sigma_{0}\right\}+\sum_{i=1}^{d} \mathrm{P}_{i}\{\tau(x)<\infty\} .
$$

Since $\lambda_{i}>\lambda_{0}$ for $i \geq 1$, and since the moment generating function of $U$ is finite in a neighborhood of $v_{0}$, the quantity $v_{i}=\sup \left\{s: \phi_{i}(s) \leq 0\right\}$ is strictly larger than $v_{0}$ for $i \geq 1$. From Lundberg's inequality, we find that

$$
\sum_{i=1}^{d} \mathrm{P}_{i}\{\tau(x)<\infty\} \leq \sum_{i=1}^{d} \mathrm{e}^{-v_{i} x}=o\left(\mathrm{e}^{-\left(v_{0}+\eta\right) x}\right)
$$

for an appropriate choice of $\eta>0$. In addition, observe that

$$
\mathrm{P}_{0}\left\{\tau(x)<\sigma_{0}\right\} \leq \mathrm{P}_{0}\{\tau(x) \leq(\hat{d}-\varepsilon) x\}+\mathrm{P}_{0}\{\infty>\tau(x)>(\hat{d}-\varepsilon) x\} \mathrm{P}\left\{\sigma_{0}>(\hat{d}-\varepsilon) x\right\}
$$

So from (11)-(12), and by substituting the bounds (15) and (14) into (13), we find that

$$
\limsup _{x \rightarrow \infty} \frac{\mathrm{P}\left\{M_{1}>x\right\}}{p_{0} \mathrm{P}\left\{\sigma_{0}>x / \phi_{0}^{\prime}\left(\nu_{0}\right)\right\}\left(-\phi_{0}^{\prime}(0) / \phi_{0}^{\prime}\left(\nu_{0}\right)\right) \mathrm{e}^{-v_{0} x}} \leq\left(\frac{\hat{d}}{\hat{d}-\varepsilon}\right)^{\alpha_{0}} .
$$

The proof of the upper bound is now completed by letting $\varepsilon \rightarrow 0$. 
We now derive the behavior of the ruin probability $\psi(x)$ as $x \rightarrow \infty$. Since $\lambda_{i}<\lambda_{0}$ for $i \geq 1$, it follows that $\phi_{i}\left(v_{0}\right)<0$ if $i \geq 1$. Consequently,

$$
\mathrm{E}\left\{\mathrm{e}^{\nu_{0} X_{1}}\right\}=\mathrm{E}\left\{\mathrm{e}^{\nu_{0} S(\sigma)}\right\}=\sum_{i=0}^{d} p_{i} \mathrm{E}\left\{\mathrm{e}^{\nu_{0} S(\sigma)} \mid L=\lambda_{i}\right\}=\sum_{i=0}^{d} p_{i} \mathrm{E}\left\{\mathrm{e}^{\phi_{i}\left(\nu_{0}\right) \sigma} \mid L=\lambda_{i}\right\}<1 .
$$

Applying Theorem 2 yields

$$
\psi(x)=\mathrm{P}\{M>x\} \sim C_{R} x^{-\alpha_{0}} l_{0}(x) \mathrm{e}^{-v_{0} x},
$$

for $C_{R}=-p_{0} \phi_{0}^{\prime}(0)\left(\phi_{0}^{\prime}\left(\nu_{0}\right)\right)^{\alpha_{0}-1} /\left(1-\mathrm{E}\left\{\mathrm{e}^{\nu_{0} S(\sigma)}\right\}\right)$, where $\mathrm{E}\left\{\mathrm{e}^{\nu_{0} S(\sigma)}\right\}$ is as given in (16).

\section{Concluding remarks}

We have examined the tail behavior of the supremum of a process of which the increment process is regenerative. We identified four different regimes, all exhibiting qualitatively different behavior for $\mathrm{P}\{M>x\}$ as $x \rightarrow \infty$. Our results focus on the zero-delayed case. It is not difficult to extend our results to the delayed case, using the representation $M=\max \left\{M_{0}, X_{0}+M_{\mathrm{zd}}\right\}$ with $M_{\mathrm{zd}}$ a random variable independent of $\left(M_{0}, X_{0}\right)$, having the same distribution of $M$ as in the zero-delayed case, where $M_{0}=\sup _{t \leq T_{0}} S(t)$ and $X_{0}=S\left(T_{0}\right)$.

\section{Acknowledgements}

The authors are indebted to Maria Vlasiou and the referee for constructive comments, which significantly improved the presentation of our results. Z. Palmowski gratefully acknowledges the support from grants NWO 613.000.310 and KBN 1P03A03128. This research would not have been possible without the hospitality of EURANDOM.

\section{References}

[1] Araman, V. F. and Glynn, P. (2006). Tail asymptotics for the maximum of perturbed random walk. Ann. Appl. Prob. 16, 1411-1431.

[2] Asmussen, S. (2003). Applied Probability and Queues, 2nd edn. Springer, New York.

[3] Asmussen, S., Schmidli, H. And Schmidt, V. (1999). Tail probabilities for non-standard risk and queueing processes with subexponential jumps. Adv. Appl. Prob. 31, 422-447.

[4] Bertoin, J. And Doney, R. (1996). Some asymptotic results for transient random walks. Adv. Appl. Prob. 28, 207-226.

[5] BJörk, T. and Grandell, J. (1988). Exponential inequalities for ruin probabilities in the Cox case. Scand. Actuarial J. 1988, 77-111.

[6] Borodin, A. N. And SAlminen, P. (1996). Handbook of Brownian Motion-Facts and Formulae. Birkhäuser, Basel.

[7] Cline, D. (1987). Convolution tails, product tails, and domains of attraction. Prob. Theory Relat. Fields 72, 529-557.

[8] DȨBicki, K., Zwart, B. And Borst, S. C. (2004). The supremum of a Gaussian process over a random interval. Statist. Prob. Lett. 68, 221-234.

[9] Denisov, D. AND ZWART, B. (2005). On a theorem of Breiman and a class of random difference equations. Submitted.

[10] Embrechts, P. and Veraverbeke, N. (1982). Estimates for the probability of ruin with special emphasis on the possibility of large claims. Insurance Math. Econom. 1, 55-72.

[11] Embrechts, P., Klüppelberg, C. And Mikosch, T. (1996). Modeling Extremal Events. Springer, Berlin.

[12] Foss, S. AND ZaCHARY, S. (2002). Asymptotics for the maximum of a modulated random walk with heavytailed increments. In Analytic Methods in Applied Probability (Amer. Math. Soc. Transl. Ser. 2), American Mathematical Society, Providence, RI, pp. 37-52. 
[13] Foss, S., Konstantopoulos, T. and Zachary, S. (2005). The principle of a big jump: discrete and continuous time modulated random walks with heavy-tailed increments. To appear in J. Theoret. Prob.

[14] Goldie, C. M. (1991). Implicit renewal theory and tails of solutions of random equations. Ann. Appl. Prob. 1, $126-166$.

[15] Grandell, J. (1991). Aspects of Risk Theory. Springer, New York.

[16] GREY, D. R. (1994). Regular variation in the tail behaviour of solutions of random difference equations. Ann. Appl. Prob. 4, 169-183.

[17] Heath, D., Resnick, S. and Samorodnitsky, G. (1998). Heavy tails and long range dependence in on/off processes and associated fluid models. Math. Operat. Res. 23, 145-165.

[18] Höglund, T. (1990). An asymptotic expression for the probability of ruin within finite time. Ann. Prob. 18, 378-389.

[19] Kella, O. ANd Whitt, W. (1992). A storage model with a two-state random environment. Operat. Res. 40, 257-262.

[20] Korshunov, D. A. (1997). On distribution tail of the maximum of a random walk. Stoch. Process. Appl. 72, 97-103.

[21] Leblanc, B., Renault, O. and Scaillet, O. (2000). A correction note on the first passage time of an OrnsteinUhlenbeck process to a boundary. Finance Stoch. 4, 109-111.

[22] Palmowski, Z. (2002). Tail probabilities for a risk process with subexponential jumps in a regenerative and diffusion environment. Prob. Math. Statist. 22, 381-405.

[23] Revuz, D. ANd Yor, M. (1999). Continuous Martingales and Brownian Motion. Springer, Berlin.

[24] Rolski, T., Schmidli, H., Schmidt, V. and Teugels, J. L. (1999). Stochastic Processes for Insurance and Finance. John Wiley, New York.

[25] Schmidul, H. (1997). An extension to the renewal theorem and an application to risk theory. Ann. Appl. Prob. 7, 121-133.

[26] Schmock, U. (1999). Estimating the value of the WinCAT coupons of the Winterthur Insurance convertible bond. ASTIN Bull. 29, 101-163.

[27] Veraverbeke, N. (1977). Asymptotic behaviour of Wiener-Hopf factors of a random walk. Stoch. Process. Appl. 5, 27-37.

[28] ZaCHARY, S. (2004). A note on Veraverbeke's theorem. Queueing Systems 46, 9-14.

[29] Zwart, B., Borst, S. C. AND Debicki, K. (2005). Subexponential asymptotics of hybrid fluid and ruin models. Ann. Appl. Prob. 15, 500-517. 